\title{
ADJunctive Ulinastatin in Sepsis Treatment in China (ADJUST study): study protocol for a randomized controlled trial
}

\author{
Wei Jiang ${ }^{1}$, Xiangyou Yư ${ }^{2}$, Tongwen $\mathrm{Sun}^{3}$, Yanfen Chai ${ }^{4}$, Ping Chang ${ }^{5}$, Zhongqing Chen ${ }^{6}$, Jingye Pan ${ }^{7}$, \\ Zhiyong Peng ${ }^{8}$, Ruilan Wang ${ }^{9}$, Xiaozhi Wang ${ }^{10}$, Yuan Xu ${ }^{11}$, Li Yu ${ }^{12}$, Qingshan Zheng ${ }^{13}$, Bin Du ${ }^{1 *}$ and for the China \\ Critical Care Clinical Trials Group (CCCCTG)
}

\begin{abstract}
Background: Sepsis is a major challenge in critical care and is associated with high mortality. Current management of sepsis and septic shock remains mainly supportive. Both basic and clinical research has shown that ulinastatin can improve the prognosis of sepsis. The aim of this trial is to evaluate the efficacy and safety profiles of ulinastatin compared with placebo.

Methods/design: In this multi-center, double-blind, randomized placebo-controlled trial we are recruiting a total of 348 subjects meeting "The Third International Consensus Definitions for Sepsis and Septic Shock" (Sepsis-3). Subjects will be randomized (1:1) to receive ulinastatin 400,000 IU three times a day for 10 days or matching placebo and usual care simultaneously. The primary outcome is 28-day all-cause mortality. Adverse events and serious adverse events will be monitored closely.

Discussion: ADJUST is a large, multi-center, double-blind, randomized, parallel-group, placebo-controlled trial of ulinastatin in mainland China and is well-designed on the basis of previous studies. The results of this trial may help to provide evidence-based recommendations for treatment of sepsis.

Trial registration: ClinicalTrials.gov, ID: NCT02647554. First registered on 27 December 2015, and last verified in December of 2016.

Protocol version: 2.1, verified on 19 July 2016.
\end{abstract}

Keywords: Ulinastatin, Sepsis, Septic shock, Randomized controlled trial, Mortality, Safety

\section{Background}

Sepsis is the leading cause of death among patients in non-coronary intensive care units (ICUs) [1]. Despite the advances in sepsis management during the past decade, the mortality rate still remains higher than $30 \%$, and up to $60 \%$ when septic shock presents [2]. In highincome countries, the population incidence rate for sepsis was 437 cases per 100,000 person-years, corresponding to a global estimate of 31.5 million cases, with potentially 5.3 million deaths annually [3]. We recently reported the standardized sepsis incidence and mortality

\footnotetext{
* Correspondence: dubin98@gmail.com

${ }^{1}$ Medical Intensive Care Unit, Peking Union Medical College Hospital, 1 Shuai Fu Yuan, Beijing 100730, China

Full list of author information is available at the end of the article
}

of 461 and 79 cases per 100,000 person-years, respectively, in China [4].

Sepsis exerts pleiotropic effects, overwhelming the host anti-inflammatory mechanisms, resulting in a generalized acute inflammatory response with life-threatening vascular, endothelial, and organ dysfunction $[5,6]$. Until recently, most research on sepsis has focused on blocking the initial dysregulated, cytokine-mediated proinflammatory response [7]. Unfortunately, numerous large clinical trials involving immunomodulatory agents in sepsis have yielded discouraging results, despite promising preliminary data from animal and preclinical studies, careful design, clearly defined inclusion criteria and thorough statistical analysis, such as recombinant platelet-activation 
factor acetylhydrolase and recombinant bactericidal/permeability-increasing protein $\left(\mathrm{rBPI}_{21}\right)$ [8-10].

Urinary trypsin inhibitor, also known as ulinastatin or bikunin, is an acid-resistant glycoprotein and Kunitztype serine protease inhibitor composed of 143 amino acid residues that can be found in human blood and urine. Ulinastatin can also prevent inflammation and cytokine-dependent signaling pathways possibly via the suppression of the phosphorylation of extracellular signal-regulated kinase $1 / 2$, c-Jun $\mathrm{N}$-terminal kinase, as well as NF- $\mathrm{kB}$ or early growth response protein-1 signaling in response to a diverse array of stresses such as oxidative stress and inflammatory stress [11-13].

In animal models of sepsis, ulinastatin could reduce both systemic and regional inflammatory responses, suppress lymphocyte apoptosis, increase production of antiinflammatory cytokines, such as interleukin (IL)-10 and IL-13, and improve survival rate [14-18]. Some pilot studies showed a trend towards decreasing mortality and duration of hospitalization with ulinastatin in severe sepsis $[19,20]$. A recent meta-analysis reported that compared with a control group, ulinastatin treatment could significantly decrease the 28-day mortality rate $(26.9 \%(152 / 565)$ vs $41.6 \%$ (228/547), RR 0.64; 95\% CI $0.54-0.75 ; P<0.01$ ); however, two out of eight enrolled trials were of low quality and some of them were combined with thymosin [21]. This meta-analysis included a recently published prospective, double-blind, multi-center randomized controlled trial (RCT) conducted in seven hospitals in India. In this trial, Karnad and colleagues [22] demonstrated that 28-day allcause mortality was significantly reduced in the ulinastatin group $(7.3 \%$ vs $20.3 \%, P=0.045)$. However, Karnad's population was at relatively lower risk, with mean age below 40 years and a mean APACHE II score $<15$ in both treated and placebo groups. The mortality in placebo group was only $20.3 \%(12 / 59)$, much lower than those in countries of similar socioeconomic status, such as $28.7 \%$ in China [23] and 55.7\% in Brazil [24]. On the other hand, there were many more cases of multi-drug-resistant infection in the placebo group (12 vs 6, placebo and ulinastatin, respectively), which might cause more deaths in the placebo group. The modified intention-to-treat (ITT) population was another problem. The primary endpoint showed significant reduction of 28-day all-cause mortality in the modified ITT population. However, in the ITT population the reduction in mortality was from $20.6 \%$ (13/ $63)$ to $10.2 \%(6 / 59)$, which was short of statistical significance $(p=0.11)$. The modification came from 4 each discontinued intervention in both arms including one and two deaths from the placebo and ulinastatin groups, respectively, and the rationale for the withdrawal of patients after randomization for early mortality was unconvincing. Their inclusion renders the trial results statistically insignificant. All these have raised concerns over the internal and external validity of the study, which significantly hampers the generalization of the study results.

We therefore designed this study to evaluate the 28-day mortality of ulinastatin treatment compared with placebo in patients with sepsis in China.

\section{Methods/design \\ Study design}

The ADJUST study is designed as a prospective, multicenter, double-blind, randomized, parallel-group, placebocontrolled (ulinastatin vs placebo 1:1) superiority trial in 12 tertiary care hospitals. The participating sites in this study include medical/surgical, medical, surgical, or emergency ICUs (see Table 1 for details of participating centers).

Patient enrollment is expected to last for up to 36 months. The end of the study is defined as the last follow-up of the last enrolled patient. The trial has been registered at ClinicalTrials.gov (NCT02647554).

\section{Recruitment}

A well-trained study coordinator in each participating center will be responsible for screening all potentially eligible patients based on the eligibility criteria. After confirmation of the patient eligibility for the trial together with the treating physician, the study coordinator will then obtain written informed consent from the patient or authorized representatives.

\section{Inclusion criteria}

Patients are eligible for the trial if they meet the criteria of "The Third International Consensus Definitions for Sepsis and Septic Shock" (Sepsis-3) and are between the age of 18 to 80 years [6]. The trial will include patients with confirmed or suspected infection and an acute change in total Sequential Organ Failure Assessment (SOFA) score $\geq 2$ points [25]. Patients will be enrolled within $48 \mathrm{~h}$ of their fulfilling Sepsis-3 criteria.

\section{Exclusion criteria}

Patients will be excluded if they meet any of the following criteria: (1) age below 18 or above 80 years; (2) pregnancy or lactation; (3) New York Heart Association Class IV chronic heart failure [26], or myocardial infarction within the previous 3 months; (4) uncontrolled blood loss; (5) cardiogenic shock; (6) advanced pulmonary fibrosis; (7) respiratory failure requiring non-invasive mechanical ventilation before enrollment; (8) severe, preexisting, parenchymal liver disease with clinically significant portal hypertension, Child-Pugh C stage cirrhosis [27], or acute liver failure [28]; (9) recipient of solid-organ or bone marrow transplant; (10) cardiopulmonary resuscitation within $72 \mathrm{~h}$ before enrollment; (11) invasive fungal infection [29, 30]; (12) active pulmonary tuberculosis 
Table 1 Research settings and names of each ethics committee

\begin{tabular}{|c|c|c|}
\hline Research setting & Ethics committee name & Approval registration number \\
\hline Peking Union Medical College Hospital & $\begin{array}{l}\text { Medical Ethics Committee of Peking Union Medical } \\
\text { College Hospital }\end{array}$ & HS-930 \\
\hline Xinjiang Medical University 1st Hospital & $\begin{array}{l}\text { Medical Ethics Committee of Xinjiang Medical } \\
\text { University } 1 \text { st Hospital }\end{array}$ & D150827-02 \\
\hline Nanfang Hospital of Southern Medical University & $\begin{array}{l}\text { Medical Ethics Committee of Nanfang Hospital } \\
\text { of Southern Medical University }\end{array}$ & NFEC-201701-K3-01 \\
\hline Zhujiang Hospital of Southern Medical University & $\begin{array}{l}\text { Medical Ethics Committee of Zhujiang Hospital } \\
\text { of Southern Medical University }\end{array}$ & 2016-ZZYXK-001 \\
\hline Tianjin Medical University General Hospital & $\begin{array}{l}\text { Medical Ethics Committee of Tianjin Medical } \\
\text { University General Hospital }\end{array}$ & IRB2017-008-01 \\
\hline Zhongnan Hospital of Wuhan University & $\begin{array}{l}\text { Medical Ethics Committee of Zhongnan } \\
\text { Hospital of Wuhan University }\end{array}$ & 药伦[2016022] \\
\hline $\begin{array}{l}\text { First People's Hospital Affiliated to Shanghai } \\
\text { Jiaotong University }\end{array}$ & $\begin{array}{l}\text { Medical Ethics Committee of First People's } \\
\text { Hospital Affiliated to Shanghai Jiaotong University }\end{array}$ & 院伦审[2016]41号 \\
\hline $\begin{array}{l}\text { the Central Hospital of Wuhan Affiliated to Tongji } \\
\text { Medical College Huazhong University of Science and } \\
\text { Technology }\end{array}$ & $\begin{array}{l}\text { Medical Ethics Committee of the Central Hospital of } \\
\text { Wuhan Affiliated to Tongji Medical College Huazhong } \\
\text { University of Science and Technology }\end{array}$ & (2016)015号 \\
\hline the First Affiliated Hospital of Zhengzhou University & $\begin{array}{l}\text { Medical Ethics Committee of the First Affiliated Hospital } \\
\text { of Zhengzhou University }\end{array}$ & 科研会审2016-49 \\
\hline Binzhou Medical University Hospital & $\begin{array}{l}\text { Medical Ethics Committee of Binzhou Medical University } \\
\text { Hospital }\end{array}$ & 2017-005-01 \\
\hline the First Affiliated Hospital of Wenzhou Medical University & $\begin{array}{l}\text { Medical Ethics Committee of the First Affiliated Hospital } \\
\text { of Wenzhou Medical University }\end{array}$ & 药伦审(2017)第012号 \\
\hline $\begin{array}{l}\text { Beijing Tsinghua Changgung Hospital Affiliated to Tsinghua } \\
\text { University }\end{array}$ & $\begin{array}{l}\text { Medical Ethics Committee of Beijing Tsinghua } \\
\text { Changgung Hospital Affiliated to Tsinghua University }\end{array}$ & $17,081-0110$ \\
\hline
\end{tabular}

[31]; (13) full-thickness thermal or chemical burn involving $30 \%$ or more of the body surface area; (14) evidence of clinically significant immunosuppression; (15) previous treatment with immunomodulatory agents, such as Xuebijing, thymosin, or intravenously administered immunoglobulin (IVIG) within 2 months before enrollment; (16) participation in an investigational clinical trial within 6 months before screening; (17) expected survival of less than 2 months or chronic vegetative state; (18) lack of commitment to full, aggressive life support; (19) history of hypersensitivity to ulinastatin. Clinically significant immunosuppression includes, but is not limited to, moderate or severe neutropenia (i.e., absolute neutrophil count < $1.0 \times 10^{9} / \mathrm{L}$ ), high-dose corticosteroids (corticosteroid equivalent of $\geq 20 \mathrm{mg}$ prednisone every day for at least 2 weeks) prior to enrollment, immunomodulatory medications (e.g., cyclosporine, azathioprine, or OKT3), chemotherapy, or radiotherapy within 2 months before enrollment, known HIV seropositivity, non-remission stage of hematological/lymphoid malignancy, and any disease sufficiently advanced to suppress resistance to infection.

\section{Study drugs, drug distribution and storage}

All study drugs and placebos are prepared by Guangdong Techpool Biopharmaceutical Co, Ltd. according to Good
Manufacturing Practice guidelines. Blinded drug (lot 031507014) or placebo (lot P031506023) are supplied as lyophilized powders in single-dose vials containing ulinastatin 100,000 IU, or inactive ingredients (i.e., mannitol, sodium chloride, and phosphate buffer), respectively. Both study drug and placebo are shipped to each participating center in cartons containing 12 vials for a 1-day supply, and stored in a cool, well-ventilated place avoiding direct sunlight.

\section{Randomization}

Following informed consent and confirmation of inclusion and exclusion criteria, participants will be randomly assigned in a 1:1 ratio to receiving either ulinastatin or placebo, with the random assignments stratified according to institution and presence of septic shock. The definition of septic shock is sepsis and hypotension requiring vasopressor support to maintain a mean blood pressure of $65 \mathrm{mmHg}$ or greater as well as having a serum lactate level greater than $2 \mathrm{mmol} / \mathrm{L}$ after adequate fluid resuscitation. Dynamic randomization will be carried out using a Distribution Annotation Interactive Web Response System through a central, secured website: https://iwrs104.drugchina.org. Minimization as the most common covariate-adaptive randomization was adopted in our trial, which derived from the method of Pocock and Simon [32]. The first subject will be 
assigned according to the simple randomization, then the allocation that produces the least total imbalance is chosen with a higher probability $(p=0.8)$ for each subject after the first one. Among the previous study the probability of $p=0.8$ has been shown to be the most efficient [33]. The reason that we used minimization in the design is that the balance of 12 centers cannot be guaranteed by the conventional randomization, and different hospitals will be considered as a covariate in the statistical analysis. The allocation is blinded to both subjects and investigators, as well as nurses and clinical research coordinators. During the treatment period, the investigator will log on to the above website every day and be given a study drug box number. With this number, the investigator could pick out the study drug box for the specific day from local storage.

The investigator will unblind allocation to the ulinastatin or placebo group should treatment of study-related adverse events (AEs) require it. The unblinding process will be well documented and reported to the principle investigator, the clinical research coordinator, and the administrative staff, when necessary. Unblinded subjects will be included in the primary, ITT analysis.

\section{Interventions}

The study group and the control group will receive ulinastatin (400,000 IU every $8 \mathrm{~h})$ and placebo, respectively, for 10 days, with the first dose administered within $8 \mathrm{~h}$ of study enrollment. In particular, the study drug is reconstituted with $10 \mathrm{ml}$ of normal saline, which will be further diluted in $100 \mathrm{ml}$ of normal saline. The study drug is administered over 1 to $2 \mathrm{~h}$ with the use of specially designed administration sets to ensure blinding. Matching placebo vials, with identical reconstitution and infusion instructions to the ulinastatin vials, will be administered on the same schedule. Apart from ulinastatin treatment, all enrolled patients will receive standard care for sepsis and septic shock as per local protocols, including, but not limited to, antimicrobial agents, intravenously administered fluids, enterally or parenterally administered nutrition, transfusion of blood and blood products, and life-sustaining therapy (e.g., vasopressors, mechanical ventilation, and renal replacement therapy). The treating physicians are encouraged to adhere to the updated Surviving Sepsis Campaign guidelines [34], although the final decision is at the discretion of the treating physicians.

Doses of ulinastatin showed significant variation in different clinical trials, from $300,000 \mathrm{IU}$ [35] to $1,200,000$ IU [36] daily. In a surveillance study of 17 ICUs of China Critical Care Clinical Trials Group (CCCCTG), 15 reported the use of ulinastatin as adjunctive treatment of sepsis, with daily dose range $200,000 \mathrm{IU}$ to $2,000,000 \mathrm{IU}$ (Bin $\mathrm{Du}$, personal communication). The safety and tolerance of multi-dose (up to $1,200,000$ IU qid) of ulinastatin have been evaluated in healthy volunteers without any serious adverse events (SAEs) [37]. In a rat model of sepsis, treatment with high-dose ulinastatin $(200,000 \mathrm{IU} / \mathrm{kg})$ significantly inhibited the production of TNF- $\alpha$, P-selectin, and thrombin-antithrombin complex compared with a low dose $(50,000 \mathrm{IU} / \mathrm{kg})$ [38]. In a previous clinical study, a high dose of ulinastatin (300,000 IU twice a day) was associated with a significantly better clinical outcome and cytokine assays compared with middle- and low-dose ulinastatin (200,000 and 100,000 IU twice daily, respectively) [39]. The dose of ulinastatin in this trial was determined by consensus based on the above evidence.

\section{Concomitant interventions}

Participants may receive stress-dose corticosteroids in the form of hydrocortisone at a daily dose of $\leq 300 \mathrm{mg}$ for the treatment of refractory septic shock. In addition, the use of non-steroidal anti-inflammatory drugs is also acceptable for the purpose of defervescence according to the local protocol. Other immunomodulatory agents (e.g., Xuebijing, thymosin, and polyclonal IVIG) should not be used during the study period, except for the purpose of treating underlying diseases (e.g., IVIG for Guillain-Barré syndrome). Use of all above drugs, if any, should be documented in the electronic Case Report Form (eCRF). No other agents with anti-inflammatory effects are allowed during the study period.

\section{Outcome measurements}

Participants will be evaluated clinically and through laboratory testing according to Fig. 1.

The following data will be recorded: demographics, sepsis diagnosis, concurrent medical conditions and comorbidities, inclusion and exclusion criteria, severity of illness and organ dysfunction scores, vital signs and laboratory results, potential confounding co-interventions (life-sustaining therapies, and use of other immunomodulatory agents), and outcomes (vital status at ICU and hospital discharge, day 28 and day 90, ICU and hospital length of stay).

\section{Primary endpoint}

The primary endpoint of this study is 28-day all-cause mortality.

\section{Secondary endpoints}

The secondary endpoints of this trial include following:

1. Mortality analysis: including 90-day all-cause mortality, ICU mortality, hospital mortality, length of stay in ICU 


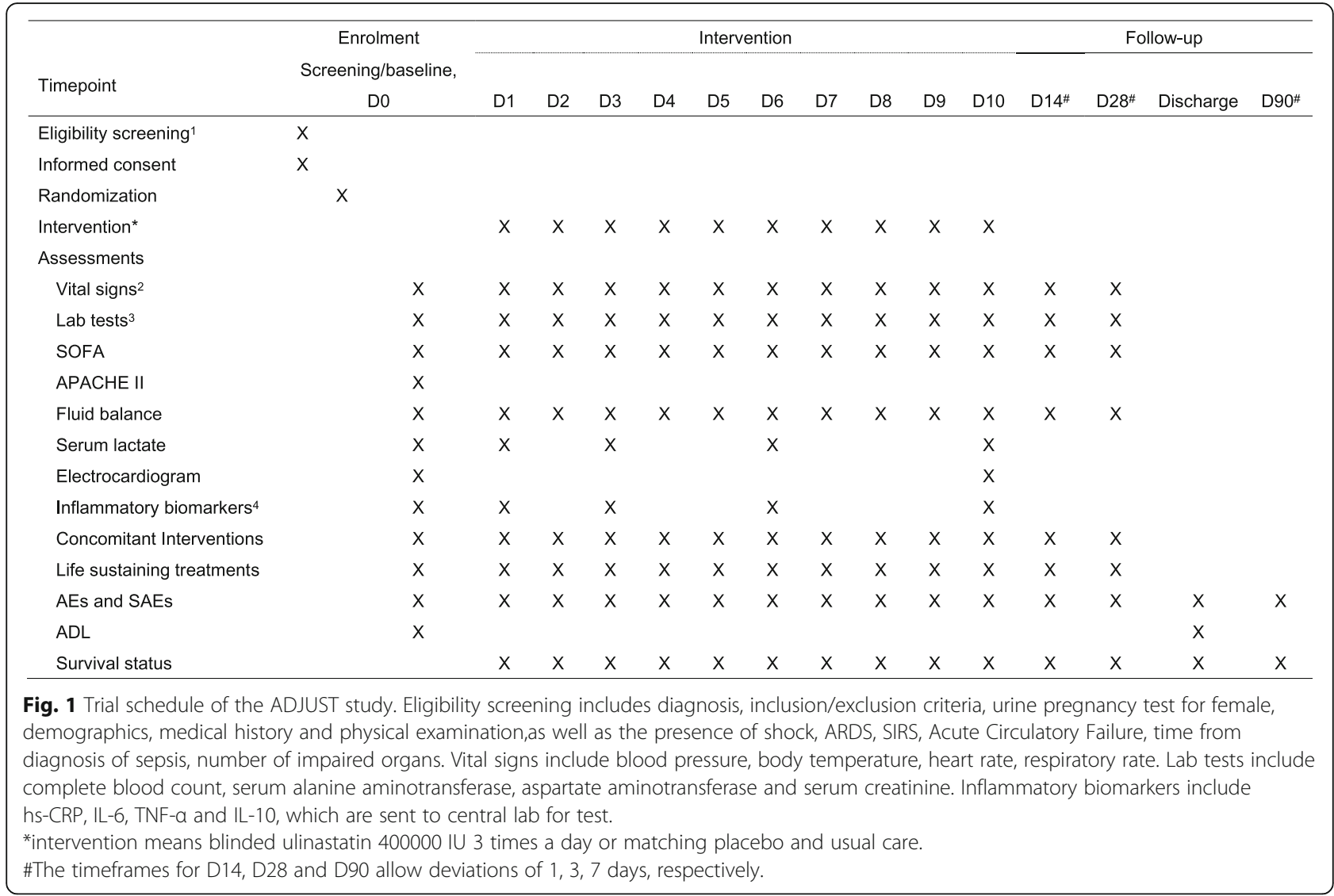

2. Organ failure and supportive intervention requirements: including SOFA scores at baseline and days $1-10,14$, and 28; use of life-sustaining therapy (i.e., vasopressor, mechanical ventilation, renal replacement therapy); lactate levels at baseline and days 1, 3, 6, and 10; fluid balance during the first 10 days

3. Inflammatory biomarkers: including high-sensitivity C-reactive protein (hs-CRP), tumor necrosis factor (TNF)- $\alpha$, IL-6, and IL-10 at baseline and days 1, 3, 6, and 10

4. Functioning: activities of daily living (ADL) level at baseline and hospital discharge [40]

We planned to collect data of organ dysfunction at baseline and various time points during the study. All the six domains of the SOFA score, including respiratory, coagulation, hepatic, cardiovascular, renal, and central nervous system, will be documented. Vasopressors, mechanical ventilation, and renal replacement therapy use will also be documented during the study as an assessment of organ dysfunction. New-onset organ dysfunction is defined as any increase of any domain in SOFA score.

Serum levels of inflammatory biomarkers (hs-CRP) and cytokines (IL-6, IL-10, and TNF- $\alpha$ ) will be measured with a chemiluminescence assay and turbidimetric inhibition immunoassay, respectively, in a central laboratory (Kingmed Center for Clinical Laboratory). All the assay chipsets were manufactured by Siemens Healthcare Diagnostics, UK.

\section{Adverse events and serious adverse events}

The safety profile of ulinastatin was an important endpoint of the current study, partially because of the relatively large dose we chose. According to the package inserts of ulinastatin for injection, adverse drug reactions are uncommon ( $0.1 \%$ to $1 \%)$, including neutropenia, eosinophilia, nausea, vomiting, diarrhea, increased alanine aminotransferase, increased aspartate aminotransferase, injection-site tenderness, flushing, pruritus, rashes, and anaphylaxis [41].

It is recognized that the patient with sepsis will experience a number of aberrations in laboratory values, signs, and symptoms due to the severity of the underlying disease and the impact of standard critical medicine therapies. These will not necessarily constitute an AE unless they are considered to be of related to study treatment or a concern in the investigator's clinical judgement. In this study, reporting of AEs will be restricted to events that are considered to be related to study treatment (possibly, probably or definitely). 
Serious adverse events are defined as any untoward medical occurrence that meets one of more of the following criteria:

- Results in death

- Is life-threatening

- Requires inpatient hospitalization or prolongation of existing hospitalization

- Results in persistent or significant disability/ incapacity

- Is a congenital anomaly/birth defect

The treating physician will be responsible for determining the causal relationship of the SAE as either definitely, probably, possibly, possibly not, or definitely not study treatment-related, as well as unclassified.

All treatment-related AEs and SAEs should be recorded on eCRF. SAEs will be reported to the Institutional Ethics Committee within $24 \mathrm{~h}$ of study staff becoming aware of the events.

The participants are provided with commercial clinical research insurance by the manufacturer of the study product.

\section{Data management}

Data management will be performed by trained staff at each participating center using the electronic data capture (EDC) system (https://edc702.drugchina.org). The quality of trial data management will be guaranteed by the reliability, access control, and traceability of the EDC system. Data collection will be restricted to those variables necessary to define baseline patient characteristics (demographics, sepsis diagnosis, concurrent medical conditions and comorbidities, inclusion and exclusion criteria, severity of illness and organ dysfunction scores, vital signs, and laboratory results), the delivery of the study drugs, potential confounding co-interventions (life-sustaining therapies, and use of other immunomodulatory agents), and outcomes (vital status at ICU and hospital discharge, day 28 , and day 90, length of stay in ICU and hospital). Randomized participants will be followed up until either death or 90 days after randomization, whichever comes first. Follow-up will be attended by study staff by either direct contact with the patient or their next of kin. Participants who withdraw from the study for any reason will be followed up according to the study follow-up schedule and analyzed on the ITT principle.

\section{Data monitoring and auditing}

An independent contract research organization (H\&J CRO International, Inc.) will be responsible for data monitoring. The quality assurance division of the same contract research organization will audit the trial regularly. This trial will be also audited by each Research Ethics Committee and China Food and Drug Administration.

\section{Statistical analysis}

\section{Sample size estimation}

We reported the hospital mortality rate of $33.5 \%$ in a 2-month prospective cohort study of 484 patients with severe sepsis/septic shock in China [23]. We estimate that 28-day all-cause mortality will be $33.5 \%$ in the control group. Treatment effect was difficult to predict. Several meta-analyses of RCTs had suggested that ulinastatin treatment was associated with an odds ratio (OR) of 0.57 (95\% CI 0.34-0.95) in hospital mortality among patients with severe sepsis/septic shock, despite the fact that the treatment effect was statistically insignificant, and all included studies were of low quality and published in the Chinese language [20, 22, 35, 42-45]. Moreover, Karnad and colleagues reported that 28-day all-cause mortality was $7.3 \%$ with ulinastatin vs $20.3 \%$ with placebo, corresponding to a RRR of 0.64 . Based on these results, we presume that a treatment effect with a RRR of 0.40 to 0.45 might be plausible estimates. We calculate that an enrollment of 348 participants will have a power of $80 \%$ to detect an absolute reduction of $14.5 \%$ (relative risk reduction of $42 \%$ ) in the study group compared to $33.5 \%$ of the placebo group, allowing for a loss to follow-up or withdrawal of $20 \%$. Considering the prevalence of severe sepsis/septic shock in our patients, and assuming the rate of patient recruitment of one to two cases per month per center (with 12 participating centers), this trial will be finished within 2 to 3 years.

\section{Data analysis}

All analyses will be performed according to the ITT principle. A $P$ value $<0.05$ is considered statistically significant. All tests are two-sided with no adjustment for multiple comparisons. Continuous variables are reported as means and standard deviations or medians and interquartile ranges. Categorical variables are reported as proportions. Pearson's chi-squared test and adjusted multivariable analysis will be applied for the primary outcome. We plan to perform subgroup analyses for the primary outcome for predefined variables: presence or absence of septic shock, time from onset of sepsis to study enrollment ( $\leq 24 \mathrm{~h}$ or 24 to $48 \mathrm{~h}$ ), and concomitant use of corticosteroids (yes or no). All the other data including age, APACHE II score, acute respiratory distress syndrome (ARDS) [46], systemic inflammatory response syndrome [47], acute circulatory failure [48], and number of failed organs will be presented as descriptive results. Pearson's chi-squared test will be used for comparison of mortality outcomes between groups. The $t$ test will be used for comparison of SOFA scores, lifesustaining interventions, lactic acid levels, fluid balance, inflammatory biomarkers, and function indicators between groups. A paired $t$ test will be used for comparison of baseline and changes during the intervention. 


\section{Interim analysis}

No interim analysis is planned for this trial.

\section{Discussion}

Ulinastatin has been evaluated as a promising drug in the treatment of sepsis, due to the inhibition of various serine proteases, inflammatory mediators produced by neutrophils, and the production of TNF- $\alpha$, IL-1, and IL-6 [49]. However, current evidence supporting the efficacy of ulinastatin in the treatment of sepsis, including RCTs, is subject to limitations such as a non-representative patient population, small sample size, and concomitant use of immunomodulatory agents (e.g., thymosin- $\alpha 1)$. As a result, the effect of ulinastatin on the mortality of sepsis warrants validation by large, wellconducted RCTs in different settings [50].

At the time of preparation of the current study, ICU patients who met the new Sepsis-3 clinical criteria were reported to have a hospital mortality rate of 16.2 to $20.2 \%$ in high-income countries (HICs) [51, 52]. Such data were still lacking in middle- and low-income countries (MLICs). Furthermore, to what extent the above results might be generalized to MLICs remains unknown. Previous studies suggested that ICU patients with sepsis in MLICs might have a much higher mortality rate than those in HICs possibly due to inadequate resources and poor quality of care. Machado and colleagues reported a hospital mortality rate up to $55.7 \%$ among Brazilian patients with severe sepsis/ septic shock [24]. In another Indian cohort, the 28-day mortality of sepsis was reported to be $62.8 \%$ [53]. As a result, using data from China [23], we estimate that 28-day all-cause mortality will be $33.5 \%$ in the control group.

The new definition of Sepsis-3 focused on organ function, so apart from the regular endpoints in clinical trials, such as mortality of various timeframes, we would like to employ more outcome measurements for organ function, together with mechanism and physical function. The four inflammatory biomarkers were chosen by the Trial Consensus Committee, and we expect that these data will add information on the mechanism of ulinastatin.

\section{Trial status}

The first subject was enrolled on 5 December 2016. The trial is recruiting.

\footnotetext{
Abbreviations

ADL: Activities of daily living; AE: Adverse event; APACHE: Acute Physiology and Chronic Health Evaluation; ARDS: Acute respiratory distress syndrome; CCCCTG: Chinese Critical Care Clinical Trials Group; eCRF: Electronic Case Report Form; EDC: Electronic data capture; HIC: High-income countries; ICU: Intensive care unit; IL: Interleukin; ITT: Intention-to-treat; IVIG: Intravenously administered immunoglobulins; MLIC: Middle- and low-income countries; RCT: Randomized controlled trial; SAE: Serious adverse event; SOFA: Sequential Organ Failure Assessment; TNF: Tumor necrosis factor
}

\section{Acknowledgements}

Not applicable.

\section{Funding}

The ADJUST trial is supported by Guangdong Techpool Biopharmaceutical Co, Ltd., which is also responsible for the supply of the study drug and placebo as well as distribution to the participating centers. Funding agency had no input into the design, conduct, data collection, statistical analysis, or writing of the manuscript.

\section{Availability of data and materials \\ Not applicable.}

\section{Confidentiality}

Each subject will be given a unique participant identification number after study enrollment. This participant identification number and quasi-identified initials will be used during randomization, study drug distribution, eCRF filling and sample transportation. The identification data, including full name, will be restricted in local medical charts and screening log, and must be preserved by researchers to maintain confidentiality.

\section{Authors' contributions}

$\mathrm{BD}$ and $X Y Y$ perceived and designed the protocol, TWS, YFC, PC, ZQC, JYP, $Z Y P, R L W, X Z W, Y X, L Y$, and QSZ participated in the design of the protocol. WJ drafted the manuscript. BD helped WJ draft and revise the manuscript. $X Y Y, T W S, Y F C, P C, Z Q C, J Y P, Z Y P, R L W, X Z W, Y X, L Y$, and WJ were involved in the data collection. WJ, BD, and QSZ analyzed the data. All authors read and approved the final manuscript.

\section{Ethics approval and consent to participate}

The protocol and the informed consent document have been reviewed and approved by the Institutional Ethics Committee of all participating centers (Table 1). Study investigators will provide potential participants with verbal and written information prior to inclusion in the study. Informed consent will be provided from participants or their authorized representatives.

\section{Consent for publication}

Not applicable. Results will be presented at relevant national and international conferences as well as being published in peer-reviewed journals.

\section{Competing interests}

The principle investigator, Professor Bin Du, will be paid a management fee for this trial according to the contract. All the other authors declare that they have no competing interests.

\section{Publisher's Note}

Springer Nature remains neutral with regard to jurisdictional claims in published maps and institutional affiliations.

\footnotetext{
Author details

${ }^{1}$ Medical Intensive Care Unit, Peking Union Medical College Hospital, 1 Shuai Fu Yuan, Beijing 100730, China. ²Department of Critical Care Medicine, Xinjiang Medical University 1st Hospital, Urumqi, Xinjiang, China. ${ }^{3}$ Department of General Intensive Care Unit, the First Affiliated Hospital of Zhengzhou University, Zhengzhou, Henan, China. ${ }^{4}$ Department of Emergency, Tianjin Medical University General Hospital, Tianjin, China. ${ }^{5}$ Department of Critical Care Medicine, Zhujiang Hospital of Southern Medical University, Guangzhou, Guangdong, China. 'Department of Critical Care Medicine, Nanfang Hospital of Southern Medical University, Guangzhou, Guangdong, China. ${ }^{7}$ Department of Critical Care Medicine, the First Affiliated Hospital of Wenzhou Medical University, Wenzhou, Zhejiang, China. ${ }^{8}$ Department of Critical Care Medicine, Zhongnan Hospital of Wuhan University, Wuhan, Hubei, China. ${ }^{9}$ Department of Critical Care Medicine, First People's Hospital Affiliated to Shanghai Jiaotong University, Shanghai, China. ${ }^{10}$ Department of Critical Care Medicine, Binzhou Medical University Hospital, Binzhou, Shandong, China. ${ }^{11}$ Department of Critical Care Medicine, Beijing Tsinghua Changgung Hospital Affiliated to Tsinghua University, Beijing, China. ${ }^{12}$ Department of Critical Care Medicine, the Central Hospital of Wuhan Affiliated to Tongji Medical College Huazhong University of Science and
} 
Technology, Wuhan, Hubei, China. ${ }^{13}$ Centre for Drug Clinical Research, Shanghai University of Traditional Chinese Medicine, Shanghai, China.

\section{Received: 4 June 2017 Accepted: 30 January 2018 Published online: 21 February 2018}

\section{References}

1. Angus DC, Linde-Zwirble WT, Lidicker J, Clermont G, Carcillo J, Pinsky MR. Epidemiology of severe sepsis in the United States: analysis of incidence, outcome, and associated costs of care. Crit Care Med. 2001;29(7):1303-10.

2. Cohen J, Vincent J-L, Adhikari NKJ, Machado FR, Angus DC, Calandra T, et al. Sepsis: a roadmap for future research. Lancet Infect Dis. 2015;15(5):581-614.

3. Fleischmann C, Scherag A, Adhikari NKJ, Hartog CS, Tsaganos T, Schlattmann $P$, et al. Assessment of global incidence and mortality of hospital-treated sepsis. Am J Resp Crit Care Med. 2016;193(3):259-72.

4. Zhou J, Tian H, Du X, Xi X, An Y, Duan M, et al. Population-based epidemiology of sepsis in a subdistrict of Beijing. Crit Care Med. 2017;45(7):1168-76.

5. Angus DC, van der Poll T. Severe sepsis and septic shock. N Engl J Med. 2013;369(9):840-51.

6. Singer M, Deutschman CS, Seymour CW, Shankar-Hari M, Annane D, Bauer $M$, et al. The Third International Consensus Definitions for Sepsis and Septic Shock (Sepsis-3). JAMA. 2016;315(8):801-10.

7. Hotchkiss RS, Monneret G, Payen D. Sepsis-induced immunosuppression: from cellular dysfunctions to immunotherapy. Nat Rev Immunol. 2013; 13(12):862-74

8. Christaki E, Anyfanti P, Opal SM. Immunomodulatory therapy for sepsis: an update. Expert Rev Anti-Infect Ther. 2011;9(11):1013-33.

9. Opal S, Laterre PF, Abraham E, Francois B, Wittebole X, Lowry S, et al. Recombinant human platelet-activating factor acetylhydrolase for treatment of severe sepsis: results of a phase III, multicenter, randomized, doubleblind, placebo-controlled, clinical trial. Crit Care Med. 2004;32(2):332-41.

10. Levin M, Quint PA, Goldstein B, Barton P, Bradley JS, Shemie SD, et al. Recombinant bactericidal/permeability-increasing protein (rBPI21) as adjunctive treatment for children with severe meningococcal sepsis: a randomised trial. Lancet. 2000:356(9234):961-7.

11. Sharony R, Yu PJ, Park J, Galloway AC, Mignatti P, Pintucci G. Protein targets of inflammatory serine proteases and cardiovascular disease. J Inflamm (Lond). 2010;7:45.

12. Shigetomi H, Onogi A, Kajiwara H, Yoshida S, Furukawa N, Haruta S, et al. Anti-inflammatory actions of serine protease inhibitors containing the Kunitz domain. Inflamm Res. 2010;59(9):679-87.

13. Inoue K-i, Takano H, Yanagisawa R, Yoshikawa T. Protective effects of urinary trypsin inhibitor on systemic inflammatory response induced by lipopolysaccharide. J Clin Biochem Nutr. 2008:43(3):139-42.

14. Wakahara K, Kobayashi H, Yagyu T, Matsuzaki H, Kondo T, Kurita N, et al. Bikunin suppresses lipopolysaccharide-induced lethality through downregulation of tumor necrosis factor-alpha and interleukin-1 beta in macrophages. J Infect Dis. 2005:191(6):930-8.

15. Ueki M, Taie S, Chujo K, Asaga T, Iwanaga Y, Ono J, et al. Urinary trypsin inhibitor reduces inflammatory response in kidney induced by lipopolysaccharide. J Biosci Bioeng. 2007;104(4):315-20.

16. Tanaka R, Fujita M, Tsuruta R, Fujimoto K, Aki HS, Kumagai $K$, et al. Urinary trypsin inhibitor suppresses excessive generation of superoxide anion radical, systemic inflammation, oxidative stress, and endothelial injury in endotoxemic rats. Inflamm Res. 2010;59(8):597-606.

17. Huang N, Wang F, Wang Y, Hou J, Li J, Deng X. Ulinastatin improves survival of septic mice by suppressing inflammatory response and lymphocyte apoptosis. J Surg Res. 2013;182(2):296-302

18. Cao YZ, Tu YY, Chen $X$, Wang BL, Zhong YX, Liu MH. Protective effect of ulinastatin against murine models of sepsis: inhibition of TNF-alpha and IL-6 and augmentation of IL-10 and IL-13. Exp Toxicol Pathol. 2012;64(6):543-7.

19. Moon SW, Lee SW, Hong YS, Park DW, Jang IJ, Yoon YH, et al. The effects of urinary trypsin inhibitor on the outcomes of severe sepsis and septic shock patients. J Korean Soc. Emerg Med. 2009;20(1):80-5.

20. Chen $S$, Chen $X$, Lai J, Yao Z. Clinical effect of ulinastatin in the treatment of septic shock. Dang Dai Yi Xue. 2012;18(18):148-50.

21. Liu D, Yu Z, Yin J, Chen Y, Zhang H, Xin F, et al. Effect of ulinastatin combined with thymosin alpha1 on sepsis: a systematic review and metaanalysis of Chinese and Indian patients. J Crit Care. 2017;39:259-66.

22. Karnad DR, Bhadade R, Verma PK, Moulick ND, Daga MK, Chafekar ND, et al. Intravenous administration of ulinastatin (human urinary trypsin inhibitor) in severe sepsis: a multicenter randomized controlled study. Intensive Care Med. 2014:40(6):830-8

23. Zhou J, Qian C, Zhao M, Yu X, Kang Y, Ma X, et al. Epidemiology and outcome of severe sepsis and septic shock in intensive care units in mainland China. PLoS One. 2014;9(9):e107181.

24. Machado FRCA, Bozza FA, Ferreira EM, Angotti Carrara FS, Sousa JL, et al. The epidemiology of sepsis in Brazilian intensive care units (the Sepsis PREvalence Assessment Database, SPREAD): an observational study. Lancet Infect Dis. 2017; 17(11):1180-9.

25. Vincent JL, Moreno R, Takala J, Willatts S, De Mendonca ABH, Reinhart CK, et al. The SOFA (sepsis-related Organ Failure Assessment) score to describe organ dysfunction/failure. On behalf of the Working Group on Sepsis-Related Problems of the European Society of Intensive Care Medicine. Intensive Care Med. 1996;22(7):707-10

26. Yancy CW, Jessup M, Bozkurt B, Butler J, Casey DE Jr, Drazner $\mathrm{MH}$, et al. 2013 ACCF/AHA guideline for the management of heart failure: a report of the American College of Cardiology Foundation/American Heart Association Task Force on practice guidelines. Circulation. 2013;128(16):e240-327.

27. Cholongitas E, Papatheodoridis GV, Vangeli M, Terreni N, Patch D, Burroughs AK. Systematic review: the model for end-stage liver disease-should it replace Child-Pugh's classification for assessing prognosis in cirrhosis? Aliment Pharm Therap. 2005;22(11-12):1079-89.

28. Liver Failure and Artificial Liver Group CSolD. Chinese Medical Association; Severe Liver Diseases and Artificial Liver Group, Chinese Society of Hepatology, Chinese Medical Association. Diagnostic and treatment guidelines for liver failure. Chin J Hepatol. 2013;21(3):177-83.

29. Pappas PG, Kauffman CA, Andes DR, Clancy CJ, Marr KA, Ostrosky-Zeichner $L$, et al. Clinical Practice Guideline for the Management of Candidiasis: 2016 Update by the Infectious Diseases Society of America. Clin Infect Dis. 2016; 62(4):e1-50.

30. Patterson TF, Thompson GR 3rd, Denning DW, Fishman JA, Hadley S, Herbrecht R, et al. Practice Guidelines for the Diagnosis and Management of Aspergillosis: 2016 Update by the Infectious Diseases Society of America. Clin Infect Dis. 2016:63(4):e1-e60.

31. Dosanjh DPS, Hinks TSC, Innes JA, Deeks JJ, Pasvol G, Hackforth S, et al. Improved diagnostic evaluation of suspected tuberculosis. Ann Intern Med. 2008;148(5):325-36.

32. Pocock SJ, Simon R. Sequential treatment assignment with balancing for prognostic factors in the controlled clinical trial. Biometrics. 1975;31(1):103-15.

33. Brown S, Thorpe H, Hawkins K, Brown J. Minimization-reducing predictability for multi-centre trials whilst retaining balance within centre. Stat Med. 2005;24(24):3715-27.

34. Rhodes A, Evans LE, Alhazzani W, Levy MM, Antonelli M, Ferrer R, et al. Surviving Sepsis Campaign: International Guidelines for Management of Sepsis and Septic Shock: 2016. Intensive Care Med. 2017:43(3):304-77.

35. Shao $Y$, Zhang $L$, Deng $L, Y a o H$. Clinical study on effects of ulinastatin on patients with systemic inflammatory response syndrome. Chin. Crit Care Med. 2005;17(4):228-30.

36. Wu C, Xiao F, Gan L, Xu Q, Yu X, Shi L, et al. Tolerance of ulinastatin in healthy volunteers. Chin J Clin Pharmacol Ther. 2007;12(1):103-6.

37. Liu Y, Wang Q, Zhan H, Jiang D, Wang Y, Li J, et al. Tolerance study of ulinastatin injection in Chinese healthy volunteers. Chin J Clin Pharmacol. 2015:31(21):2111-3.

38. Liu Y, Wu X. Effect of ulinastatin on serum levels of tumor necrosis factor-alpha P-selectin, and thrombin-antithrombin complex in young rats with sepsis. Chin J Contemp Pediatr. 2017;19(2):237-41.

39. Liang Y. Clinical efficacy of various doses of ulinastatin in patients with severe sepsis. He Bei Yi Yao. 2013;35(14):2116-7.

40. Mahoney FI, Barthel DW. Functional evaluation: the Barthel Index. Md State Med J. 1965:14:61-5.

41. Package insert of ulinastatin for injection. Available from: https://wenku. baidu.com/view/b4111b09bb68a98271fefaf4.html. Accessed 23 Jan 2018.

42. Li J, Lai J, Jiang Y, Yao Z, Chen X, Chen S. Study of ulinastatin against gastrointestinal complications in patients with septic shock. Xian Dai Yi Xue. 2010;38(3):263-5.

43. Li J, Lai J, Liu C, Yao Z, Chen S, Chen X, et al. Clinical study of ulinastatin on lung injury in patients with septic shock. Xian Dai Yi Xue. 2011;39(1):41-4.

44. Wang $\mathrm{L}$, Zhong $\mathrm{H}$, Zhang J. Clinical effects of ulinastatin in 90 patients with septic shock. Zhong Guo Bao Jian Ying Yang. 2013;1(1):222.

45. Feng $L$, Zhou Q. Meta-analysis of the efficacy of the septic shock by therapy of ulinastatin. Chin J Clinicians (electronic edition). 2013;7(9):3952-6. 
46. The ARDS Definition Task Force. Acute respiratory distress syndrome: the Berlin Definition. JAMA. 2012;307(23):2526-33.

47. Bone RC, Balk RA, Cerra FB, Dellinger RP, Fein AM, Knaus WA, et al. Definitions for sepsis and organ failure and guidelines for the use of innovative therapies in sepsis. Chest. 1992;101(6):1644-55.

48. Association EMBoCMD. Expert consensus on acute circulatory failure: clinical practice of Chinese emergency physicians. Chin J Emerg Med. 2016;25(2):143-9.

49. Lim YP, Bendelja K, Opal SM, Siryaporn E, Hixson DC, Palardy JE. Correlation between mortality and the levels of inter-alpha inhibitors in the plasma of patients with severe sepsis. J Infect Dis. 2003;188(6):919-26.

50. Linder A, Russell JA. An exciting candidate therapy for sepsis: ulinastatin, a urinary protease inhibitor. Intensive Care Med. 2014;40(8):1164-7.

51. Seymour CW, Liu VX, Iwashyna TJ, Brunkhorst FM, Rea TD, Scherag A, et al. Assessment of clinical criteria for sepsis: for the Third International Consensus Definitions for Sepsis and Septic Shock (Sepsis-3). JAMA. 2016; 315(8):762-74.

52. Raith EP, Udy AA, Bailey M, McGloughlin S, Maclsaac C, Bellomo R, et al. Prognostic Accuracy of the SOFA score, SIRS criteria, and qSOFA score for in-hospital mortality among adults with suspected infection admitted to the intensive care unit. JAMA. 2017;317(3):290-300.

53. Chatterjee S, Bhattacharya M, Todi SK. Epidemiology of adult-population sepsis in India: a single center 5 year experience. Indian J Crit Care Med. 2017;21(9):573-7.

\section{Submit your next manuscript to BioMed Central} and we will help you at every step:

- We accept pre-submission inquiries

- Our selector tool helps you to find the most relevant journal

- We provide round the clock customer support

- Convenient online submission

- Thorough peer review

- Inclusion in PubMed and all major indexing services

- Maximum visibility for your research

Submit your manuscript at www.biomedcentral.com/submit 